\title{
ON NÖRLUND SUMMATION AND ERGODIC THEORY, WITH APPLICATIONS TO POWER SERIES OF HILBERT CONTRACTIONS.
}

\author{
CHRISTOPHE CUNY AND MICHEL WEBER
}

\begin{abstract}
We show that if $\mathbf{a}=\left(a_{n}\right)_{n \in \mathbb{N}}$ is a good weight for the dominated weighted ergodic theorem in $L^{p}, p>1$, then the Nörlund matrix $N_{\mathbf{a}}=\left\{a_{i-j} / A_{i}\right\}_{0 \leq j \leq i}, A_{i}=\sum_{k=0}^{i}\left|a_{k}\right|$ is bounded on $\ell^{p}(\mathbb{N})$. We study the regularity (convergence in norm, almost everywhere) of operators in ergodic theory: power series of Hilbert contractions, and power series $\sum_{n \in \mathbb{N}} a_{n} P_{n} f$ of $L^{2}$-contractions, and establish similar tight relations with the Nörlund operator associated to the modulus coefficient sequence $\left(\left|a_{n}\right|\right)_{n \in \mathbb{N}}$.
\end{abstract}

\section{Introduction}

Let $\mathbf{a}:=\left(a_{n}\right)_{n \in \mathbb{N}}$ be a sequence of complex or real numbers (we take the convention $\mathbb{N}=$ $\{0,1,2, \ldots\}$. We associate with $\mathbf{a}$ an infinite matrix $N_{\mathbf{a}}=\left(a_{i j}\right)_{i, j \in \mathbb{N}}$, called a Nörlund matrix, in the following way. For every $i \geq 0$, set $A_{i}:=\sum_{k=0}^{i}\left|a_{k}\right|$. We then define

$$
\begin{cases}a_{i j}:=a_{i-j} / A_{i} & \text { if } 0 \leq j \leq i \text { and } A_{i}>0, \\ a_{i j}:=0 & \text { if } j>i \text { or } A_{i}=0 .\end{cases}
$$

Some authors consider instead $A_{i}=\sum_{k=0}^{i} a_{k}$, assuming then that it does not vanish.

Then $N_{\mathbf{a}}$ induces naturally a (possibly unbounded) operator on $\ell^{p}(\mathbb{N})$ for any $p \geq 1$. The matter of deciding whether this operator is bounded on some (or any) $\ell^{p}(\mathbb{N})$ is far from being solved. As noted by Bennett 4, it seems, so far, that the best general known condition guaranteeing that $N_{\mathbf{a}}$ is bounded on any $\ell^{p}(\mathbb{N}), p>1$, is that $a_{n}=O\left(A_{n} / n\right)$, see for instance Borwein and Cass [6]. That condition is realized when, for instance $\left(a_{n}\right)_{n \in \mathbb{N}}$ is a non-increasing sequence of positive numbers.

Let $c_{00}:=\left\{\left(u_{n}\right)_{n \in \mathbb{N}} \in \mathbb{C}^{\mathbb{N}}: \exists n_{0} \in \mathbb{N} / u_{n}=0, \forall n \geq n_{0}\right\}$. Recall that the boundedness of $N_{\mathbf{a}}$ on $\ell^{p}, p>1$, means that there exists $C_{p}>0$ such that, for any sequence $\left(u_{n}\right)_{n \in \mathbb{N}} \in c_{00}$,

$$
\sum_{i \geq 0}\left|\frac{1}{A_{i}} \sum_{j=0}^{i} a_{i-j} u_{j}\right|^{p} \leq C_{p}^{p} \sum_{i \geq 0}\left|u_{i}\right|^{p} .
$$

Equivalently, we have the dual formulation: for any sequence $\left(v_{n}\right)_{n \in \mathbb{N}} \in c_{00}, q=p /(p-1)$,

$$
\sum_{j \geq 0}\left|\sum_{i \geq j} a_{i-j} v_{i} / A_{i}\right|^{q} \leq C_{p}^{q} \sum_{j \geq 0}\left|v_{j}\right|^{q}
$$

2010 Mathematics Subject Classification. 47A35, 47D37. 
The latter is easily seen to be also equivalent to: for any sequence $\left(v_{n}\right)_{n \in \mathbb{N}} \in c_{00}$,

$$
\sum_{j \geq 0}\left|\sum_{i \geq j} a_{i-j} v_{i}\right|^{q}=\sum_{j \geq 0}\left|\sum_{i \geq 0} a_{i} v_{i+j}\right|^{q} \leq C_{p}^{q} \sum_{j \geq 0}\left|A_{j} v_{j}\right|^{q} .
$$

Moreover, it follows from (3) that

$$
\sum_{i \geq 0} \frac{\left|a_{i}\right|^{p}}{A_{i}^{p}} \leq C_{p}^{p}
$$

where $a_{i} / A_{i}$ has to be interpreted as 0 when $A_{i}=0$.

We show that Nörlund matrices are connected with two different topics from ergodic theory. We establish tight relations between regularity (convergence in norm, almost everywhere) of operators in ergodic theory (power series of Hilbert contractions, power series of $L^{2}$-contractions, dominated weighted ergodic theorems, and naturally associated Nörlund matrices. We obtain conditions ensuring norm convergence of power series of Hilbert contractions, and also almost everywhere convergence of power series $\sum_{n \in \mathbb{N}} a_{n} P_{n} f$ of $L^{2}$-contractions. These conditions are expressed in terms of the Nörlund operator associated to the modulus coefficient sequence $\left(\left|a_{n}\right|\right)_{n \in \mathbb{N}}$.

\section{Nörlund matrices and dominated weighted ergodic theorems}

We first observe a connection between Nörlund matrices and dominated weighted ergodic theorems.

We say that a sequence $\left(a_{n}\right)_{n \in \mathbb{N}}$, of complex numbers, is good for the dominated weighted ergodic theorem in $L^{p}, p>1$, if there exists $C>0$ such that for every dynamical system $(X, \Sigma, \nu, \tau)$, writing $A_{n}:=\sum_{k=0}^{n}\left|a_{k}\right|$, we have

$$
\left\|\sup _{n \geq 0} \frac{1}{A_{n}}\left|\sum_{k=0}^{n} a_{k} f \circ \tau^{k}\right|\right\|_{L^{p}(\nu)} \leq C\|f\|_{L^{p}(\nu)} \quad \forall f \in L^{p}(\nu) .
$$

Here again we take the convention that $\frac{1}{A_{n}}\left|\sum_{k=0}^{n} a_{k} f \circ \tau^{k}\right|=0$ if $A_{n}=0$.

The next lemma is well-known, it is in the spirit of the so-called Conze principle, see for instance [29, Th. 5.4.3]. It states a converse of Calderon's transference principle.

Lemma 1. Let $\left(a_{n}\right)_{n \in \mathbb{N}}$ be good for the dominated weighted ergodic theorem in $L^{p}, p>1$. Then, with the best constant $C>0$ appearing in (6), we have for every $\left(v_{n}\right)_{n \in \mathbb{Z}} \in \ell^{p}(\mathbb{Z})$,

$$
\sum_{i \in \mathbb{Z}}\left(\sup _{n \geq 0} \frac{1}{A_{n}}\left|\sum_{j=0}^{n} a_{j} v_{i+j}\right|\right)^{p} \leq C^{p} \sum_{i \in \mathbb{Z}}\left|v_{j}\right|^{p} .
$$

Proof. Let $\left(v_{n}\right)_{n \in \mathbb{Z}} \in \ell^{p}(\mathbb{Z})$. Let $N>M \geq 1$ be integers (one has in mind that $N \gg M$ ). Take $X=\{-N,-N+1, \ldots, N-1, N\}, \nu:=\frac{1}{2 N+1} \sum_{k=-N}^{N} \delta_{k}$ and $\tau$ the transformation given by $\theta(k)=k+1$ if $k \neq N$ and $\theta(N)=-N$. Define $f$ on $X$, by $f(k)=v_{k}$ for every $k \in X$. By (6), we have

$$
\begin{gathered}
\frac{1}{2 N+1} \sum_{i=-N}^{N+1-M}\left(\sup _{0 \leq m \leq M} \frac{1}{A_{m}}\left|\sum_{k=0}^{m} a_{k} v_{i+k}\right|\right)^{p} \leq\left\|\sup _{n \geq 0} \frac{1}{A_{n}}\left|\sum_{k=0}^{n} a_{k} f \circ \tau^{k}\right|\right\|_{L^{p}(\nu)}^{p} \\
\leq C^{p}\|f\|_{L^{p}(\nu)}^{p}=\frac{C^{p}}{2 N+1} \sum_{i=-N}^{N}\left|v_{i}\right|^{p} .
\end{gathered}
$$


Multiplying (8) by $2 N+1$, letting first $N \rightarrow+\infty$ and then $M \rightarrow+\infty$, we derive (77).

Remark. Our proof is based on the use of the dominated weighted ergodic theorem on periodic systems (the rotations on $\mathbb{Z} /(2 N+1) \mathbb{Z})$. To give a proof based on the dominated weighted ergodic theorem on a single (but ergodic and non-atomic) dynamical system, one could use Rohlin's lemma (see for instance Weber [29, p. 270] for a statement of the lemma).

We deduce the following.

Proposition 2. Let $\mathbf{a}=\left(a_{n}\right)_{n \in \mathbb{N}}$ be a good weight for the dominated weighted ergodic theorem in $L^{p}, p>1$. Then, the Nörlund matrix $N_{\mathbf{a}}$ is bounded on $\ell^{p}(\mathbb{N})$. Moreover, for every nonincreasing sequence of nonnegative numbers $\left(b_{n}\right)_{n \in \mathbb{N}}$, writing $\mathbf{c}:=\left(a_{n} b_{n}\right)_{n \in \mathbb{N}}, N_{\mathbf{c}}$ is bounded on $\ell^{p}(\mathbb{N})$.

Remark. It is unclear whether " $N_{\mathbf{a}}$ bounded on $\ell^{p}$ " implies " $N_{\mathbf{c}}$ bounded on $\ell^{p}$ ", in general. Proof. Let $\left(u_{n}\right)_{n \in \mathbb{N}} \in \ell^{p}(\mathbb{N})$. Define $\left(v_{n}\right) \in \ell^{p}(\mathbb{Z})$ as follows. $v_{n}:=u_{-n}$ if $n \leq 0$ and $v_{n}:=0$ if $n>0$. Using (7) and for every $i \geq 1$ the trivial estimate

$$
\frac{1}{A_{i}}\left|\sum_{j=0}^{i} a_{j} v_{-i+j}\right| \leq \sup _{n \geq 0} \frac{1}{A_{n}}\left|\sum_{j=0}^{n} a_{j} v_{-i+j}\right|,
$$

we infer that

$$
\sum_{i \geq 0}\left(\frac{1}{A_{i}}\left|\sum_{j=0}^{i} a_{j} v_{-i+j}\right|\right)^{p} \leq C^{p} \sum_{i \in \mathbb{Z}}\left|v_{i}\right|^{p}=C^{p} \sum_{i \geq 0}\left|u_{i}\right|^{p} .
$$

Using that $v_{-i+j}=u_{i-j}$ when $j \leq i$, we derive that $N_{\mathbf{a}}$ is bounded on $\ell^{p}$.

To prove the last assertion, one just has to notice that, using Abel summation, $\left(c_{n}\right)_{n \in \mathbb{N}}$ is a good weight for the dominated weighted ergodic theorem.

Of course, as one can see from the above proof, the fact that $\mathbf{a}=\left(a_{n}\right)_{n \in \mathbb{N}}$ be a good weight for the dominated weighted ergodic theorem in $L^{p}$ is a much stronger statement than the fact that $N_{\mathrm{a}}$ be bounded on $\ell^{p}(\mathbb{N})$. Hence, Proposition 2 should not be seen as a method to prove boundedness of some Nörlund matrices, but as a source of examples of Nörlund matrices, since there are many examples of sequences that are known to be good for the dominated weighted ergodic theorem. We provide some of them below. One may also consult the survey by Bellow and Losert [3] for dominated weighted ergodic theorems with bounded weights. More arithmetical sequences may be found in Cuny and Weber [17].

Examples. The following sequences $\left(a_{n}\right)_{n \in \mathbb{N}}$ are good for the dominated weighted ergodic theorem in $L^{p}$, for every $p>1$ :

(i) (Bourgain and Wierdl, [7, 30]) Let $\mathcal{P}$ be the set of prime numbers and take $a_{n}:=\delta_{\mathcal{P}}(n)$, for every $n \in \mathbb{N}$.

(ii) (Bourgain, 9]) Let $\mathcal{S}$ be the set of squares and take $a_{n}:=\delta_{\mathcal{S}}(n)$, for every $n \in \mathbb{N}$.

(iii) (Cuny and Weber, 17]) Take $a_{0}=0$ and for every $n \in \mathbb{N}$ take $a_{n}=d_{n}$, the number of divisors of $n$.

We now give an example which does not work on every $L^{p}, p>1$. Let $(X, \Sigma, \mu, \theta)$ be an ergodic dynamical system. Let $g \in L^{q}(\mu)$, for some $1<q \leq \infty$.

(iv) (Bourgain, Demeter, Lacey, Tao and Thiele, [8], 21] and [19]) There exists $\bar{X} \in \Sigma$ with $\mu(\bar{X})=1$ such that for every $x \in \bar{X},\left(a_{n}\right)_{n \in \mathbb{N}}:=\left(g \circ \theta^{n}(x)\right)_{n \in \mathbb{N}}$ is good for the dominated weighted ergodic theorem in $L^{p}$ for every $p>1$ such that $1 / p+1 / q<3 / 2$. 
Let us notice that none of the above examples satisfies the previously mentionned criterium: $\sup _{n \in \mathbb{N}} n\left|a_{n}\right| / A_{n}<\infty$. The fact that the Nörlund matrix associated with the sequence $\left(a_{n}\right)_{n \in \mathbb{N}}$ in example (ii) is bounded has been proved by Borwein [5].

\section{Norm convergence of power series of Hilbert contractions}

Let $P$ be a contraction of a (real or complex) Hilbert space $\mathcal{H}$. Given a sequence of complex numbers $\left(a_{n}\right)_{n \in \mathbb{N}}$ and $f \in \mathcal{H}$, we are interested in finding conditions involving $\left(\left\|P^{n} f\right\|_{\mathcal{H}}\right)_{n \in \mathbb{N}}$ sufficient for the norm convergence of $\sum_{n \in \mathbb{N}} a_{n} P^{n} f$.

An obvious condition is the following

$$
\sum_{n \in \mathbb{N}}\left|a_{n}\right|\left\|P^{n} f\right\|_{\mathcal{H}}<\infty .
$$

Sufficient conditions involving $\left(\left\|f+\ldots+P^{n} f\right\|_{\mathcal{H}}\right)_{n \in \mathbb{N}}$ have been obtained when $P$ is unitary (i.e. $P^{*}=P^{-1}$ ) or, more generally, normal (i.e. $P P^{*}=P^{*} P$ ), if moreover $\left(a_{n}\right)_{n \in \mathbb{N}}$ is regular (at least nonnegative and nonincreasing). Let us mention the papers [23] and [13, see also [14] for some $L^{p}$ versions.

Recall that, see for instance Nagy and Foias 25] (see also Shäffer [?] for an explicit matrix construction), $P$ admits a unitary dilation, that is, there exist another Hilbert space $\mathcal{K}$, with $\mathcal{H} \subset \mathcal{K}$, and a unitary operator $U$ on $\mathcal{K}$ such that $E U^{n}=P^{n}$ for every $n \geq 1$, where $E$ is the orthogonal projection onto $\mathcal{H}$.

We start with some simple lemmas. The first one appears in Cuny and Lin [16, but we recall the short proof.

Lemma 3. For every $n \in \mathbb{N}$ and every $\ell \geq 1$, the spaces $\left(U^{-n} P^{n}-U^{-n-1} P^{n+1}\right) \mathcal{H}$ and $U^{-n-\ell} P^{n+\ell} \mathcal{H}$ are orthogonal (in $\mathcal{K}$ ).

Proof. Let $f, g \in \mathcal{H}$. Let $n \in \mathbb{N}$ and $\ell \geq 1$. We have

$$
\begin{gathered}
\left\langle\left(U^{-n} P^{n}-U^{-n-1} P^{n+1}\right) f, U^{-n-\ell} P^{n+\ell} g\right\rangle_{\mathcal{K}}=\left\langle U^{\ell} P^{n} f, P^{n+\ell} g\right\rangle_{\mathcal{K}}-\left\langle U^{\ell-1} P^{n+1} f, P^{n+\ell} g\right\rangle_{\mathcal{K}} \\
=\left\langle P^{n+\ell} f, P^{n+\ell} g\right\rangle_{\mathcal{K}}-\left\langle P^{n+\ell} f, P^{n+\ell} g\right\rangle_{\mathcal{K}}=0 .
\end{gathered}
$$

Lemma 4. Let $f \in \mathcal{H}$ be such that $\left\|P^{m} f\right\|_{\mathcal{H}} \rightarrow 0$ as $m \rightarrow+\infty$. Then, for every $n \geq 1$, $\left\|P^{n} f\right\|_{\mathcal{H}}^{2}=\left\|P^{n} f\right\|_{\mathcal{K}}^{2}=\sum_{k \geq n}\left\|U^{-k} P^{k} f-U^{-k-1} P^{k+1}\right\|_{\mathcal{K}}^{2}$. In particular, for any positive and non-decreasing sequence $\left(b_{n}\right)_{n \geq 0}$, the following are equivalent (setting $b_{-1}=0$ ).

(i) $\sum_{n \in \mathbb{N}}\left(b_{n}-b_{n-1}\right)\left\|P^{n} f\right\|_{\mathcal{H}}^{2}<\infty$;

(ii) $\sum_{n \in \mathbb{N}} b_{n}\left\|U^{-n} P^{n} f-U^{-n-1} P^{n+1} f\right\|_{\mathcal{K}}^{2}<\infty$.

Remarks. Notice that by Kronecker's lemma, if $(i)$ holds $\left\|P^{n} f\right\|_{\mathcal{H}}^{2} \sum_{k=0}^{n}\left(b_{k+1}-b_{k}\right)=\left\|P^{n} f\right\|_{\mathcal{H}}^{2}\left(b_{n+1}-\right.$ $\left.b_{0}\right) \rightarrow 0$ as $n \rightarrow+\infty$. In particular, since $\left(b_{n}\right)_{n \in \mathbb{N}}$ is non decreasing, $\left\|P^{n} f\right\|_{\mathcal{H}} \rightarrow 0$. Item $(i)$ is satisfied if $\sum_{n \geq 0} b_{2^{n+1}}\left\|P^{2^{n}} f\right\|_{\mathcal{H}}^{2}<\infty$.

Proof. Since $\left\|P^{n} f\right\| \rightarrow 0$, for every $n \in \mathbb{N}$, we have, (with convergence in $\mathcal{K}$ )

$$
P^{n} f=\sum_{k \geq 0}\left(U^{-k} P^{n+k} f-U^{-k-1} P^{n+k+1} f\right) .
$$


By the above lemma the terms of that series lie in orthogonal spaces. Hence,

$$
\begin{aligned}
\left\|P^{n} f\right\|_{\mathcal{K}}^{2} & =\sum_{k \geq 0}\left\|U^{-k} P^{n+k} f-U^{-k-1} P^{n+k+1}\right\|_{\mathcal{K}}^{2} \\
& =\sum_{k \geq n}\left\|U^{-k} P^{k} f-U^{-k-1} P^{k+1}\right\|_{\mathcal{K}}^{2},
\end{aligned}
$$

where we used that $U$ is unitary (and a change of variable) for the last identity. Then, the equivalence of $(i)$ and $(i i)$ follows by Fubini.

Given a sequence of complex numbers $\left(a_{n}\right)_{n \in \mathbb{N}}$, consider the following conditions

$$
\begin{gathered}
\sum_{n \in \mathbb{N}}\left|a_{n}\right|\left(\sum_{k=0}^{n}\left|a_{k}\right|\right)\left\|P^{n} f\right\|_{\mathcal{H}}^{2}<\infty, \\
\sum_{n \in \mathbb{N}}\left(\sum_{k=0}^{n}\left|a_{k}\right|\right)^{2}\left\|U^{-n} P^{n} f-U^{-n-1} P^{n+1} f\right\|_{\mathcal{K}}^{2}<\infty
\end{gathered}
$$

By Lemma (41), when $\left\|P^{n} f\right\|_{\mathcal{H}} \rightarrow 0$, (11) and (12) are equivalent. Assume that (91) holds. Then, since $\left(\left\|P^{n} f\right\|_{\mathcal{H}}\right)_{n \in \mathbb{N}}$ is nonincreasing, $\sup _{n \in \mathbb{N}}\left\|P^{n} f\right\| \sum_{k=0}^{n}\left|a_{k}\right|<\infty$ and (11) holds. Hence, (11) is always weaker than (9).

Proposition 5. Let $\left(a_{n}\right)_{n \in \mathbb{N}} \in \mathbb{C}^{\mathbb{N}}$ be such that $N_{|\mathbf{a}|}$ be bounded on $\ell^{2}(\mathbb{N})$ where $|\mathbf{a}|=\left(a_{n}\right)_{n \in \mathbb{N}}$. Let $f \in \mathcal{H}$ be such that either of conditions (111) or (12) hold. Then, the series $\sum_{n \in \mathbb{N}} a_{n} P^{n} f$ converges in $\mathcal{H}$.

Proof. Since $N_{|\mathbf{a}|}$ is bounded on $\ell^{2}(\mathbb{N})$, then by (5) (with $p=2$ )

$$
\sum_{n \in \mathbb{N}} \frac{a_{n}^{2}}{A_{n}^{2}}<\infty .
$$

Let $q>p \geq 1$ be integers and write $V_{p, q} f:=\sum_{k=p}^{q} a_{k} P^{k} f$. For every $n \in \mathbb{N}$, let $u_{n}:=$ $\left\|U^{-n} P^{n} f-U^{-n-1} P^{n+1} f\right\|_{\mathcal{K}}$ and $v_{n}:=A_{n} u_{n}$, where $A_{n}=\sum_{k=0}^{n}\left|a_{k}\right|$. Finally, let $\mathbf{v}:=\left(v_{n}\right)_{n \in \mathbb{N}}$. By Lemma 4 and using that $U$ is unitary, we have

$$
\left\|V_{p, q} f\right\|_{\mathcal{K}}^{2}=\sum_{n \in \mathbb{N}}\left\|U^{-n} P^{n} V_{p, q} f-U^{-n-1} P^{n+1} V_{p, q} f\right\|_{\mathcal{K}}^{2} \leq \sum_{n \in \mathbb{N}}\left(\sum_{k=p}^{q}\left|a_{k}\right| u_{n+k}\right)^{2} .
$$

By Cauchy's criteria one has to prove that $\left\|V_{p, q} f\right\|_{\mathcal{K}} \rightarrow 0$ as $p, q \rightarrow+\infty$. Using the Lebesgue dominated theorem for the counting measure on $\mathbb{N}$, it suffices to prove that

$$
\sum_{k=p}^{q}\left|a_{k}\right| u_{n+k} \underset{p, q \rightarrow+\infty}{\longrightarrow} 0
$$

and that

$$
\sum_{n \in \mathbb{N}}\left(\sum_{k \geq 0}\left|a_{k}\right| u_{n+k}\right)^{2}
$$

The convergence (15) follows from Cauchy-Schwarz combined with the assumed condition (12) and (13). 
FTo prove (16), it suffices to notice that

$$
\begin{gathered}
\sum_{n \in \mathbb{N}}\left(\sum_{k \geq 0}\left|a_{k}\right| u_{n+k}\right)^{2} \leq \sum_{n \in \mathbb{N}}\left(\sum_{k \geq n}\left|a_{k-n}\right| u_{k}\right)^{2}=\left\|N_{|\mathbf{a}|}^{*} \mathbf{v}\right\|_{\ell^{2}(\mathbb{N})}^{2} \\
\leq\left\|N_{|\mathbf{a}|}^{*}\right\|^{2}\|\mathbf{v}\|_{\ell^{2}(\mathbb{N})}^{2}=\left\|N_{|\mathbf{a}|}\right\|^{2} \sum_{n \in \mathbb{N}} A_{n}^{2} u_{n}^{2} .
\end{gathered}
$$

The proposition has been proved in 16 in the case where $a_{n}=n^{-1 / 2}$. An important case corresponds to the situation where $a_{n}=1$ for every $n \in \mathbb{N}$. Then, the proposition gives a sufficient condition (namely $\sum_{n \in \mathbb{N}} n\left\|P^{n} f\right\|_{\mathcal{H}}^{2}<\infty$ ) for $f$ to be a coboundary (i.e. $f=(I-P) g$ for some $g \in \mathcal{H}$ ). This sufficient condition has been obtained independently by Volný 28 in the special case where $P$ is a Markov operator on $L^{2}(m)$. His proof (which does not appeal to the notion of Nörlund matrices) is essentially the same, since the shift on the space of trajectories of the associated Markov chain plays the role of the unitary dilation.

Proposition 6. Let $\left(a_{n}\right)_{n \in \mathbb{N}} \in \mathbb{C}^{\mathbb{N}}$. Assume that for every contraction $P$ on a Hilbert space $\mathcal{H}$ the following property holds : "If (11) holds for some $f \in \mathcal{H}$ then $\sum_{n \in \mathbb{N}} a_{n} P^{n} f$ converges in $\mathcal{H}$ ". Then, $N_{\mathbf{a}}$ is bounded on $\ell^{2}$.

Proof. Let $P$ be a contraction on a Hilbert space $\mathcal{H}$ satisfying the above property. Let $\mathcal{L}:=\{f \in$ $\mathcal{H}: \sum_{n \in \mathbb{N}}\left|a_{n}\right|\left(\sum_{k=0}^{n}\left|a_{k}\right|\right)\left\|P^{n} f\right\|_{\mathcal{H}}^{2}<\infty$. Then, $\mathcal{L}$ is a Hilbert space and we define an operator $T$ on $\mathcal{L}$, by setting $T f=\sum_{n \in \mathbb{N}} a_{n} P^{n} f$ for every $f \in \mathcal{L}$. Then, by the Banach-Steinhaus, theorem $T$ is continuous. Hence, there exists $C=C_{\mathcal{H}, P}$, such that $\left\|\sum_{n \in \mathbb{N}} a_{n} P^{n} f\right\|_{\mathcal{H}} \leq C\left(\sum_{n \in \mathbb{N}}\left(b_{n+1}-\right.\right.$ $\left.\left.b_{n}\right)\left\|P^{n} f\right\|_{\mathcal{H}}^{2}\right)^{1 / 2}<\infty$.

Let us prove the proposition. We give a probabilistic proof. Let $(\Omega, \mathcal{F}, \mathbb{P})$ be the probability space given by $\Omega=\{-1,1\}^{\mathbb{Z}}, \mathcal{F}$ the product $\sigma$-algebra and $\mathbb{P}=\mu^{\otimes \mathbb{Z}}$, with $\mu(0)=\mu(1)=1 / 2$. Let $\theta$ be the shift on $\Omega$ and $\left(\varepsilon_{n}\right)_{n \in \mathbb{Z}}$ be the coordinate process. In particular, $\varepsilon_{n+1}=\varepsilon_{n} \circ \theta$ and $\left(\varepsilon_{n}\right)_{n \in \mathbb{Z}}$ is iid.

Denote $\mathcal{F}_{0}:=\sigma\left\{\varepsilon_{i}, i \leq 0\right\}$. Set $\mathcal{H}:=L^{2}\left(\Omega, \mathcal{F}_{0}, \mathbb{P}\right)$ and $\mathcal{K}:=L^{2}(\Omega, \mathcal{F}, \mathbb{P})$ and define two operators $U$ and $P$ on $\mathcal{K}$ and $\mathcal{H}$ respectively by $U f=f \circ \theta$ for every $f \in \mathcal{K}$ and $P f=\mathbb{E}\left(f \circ \theta \mid \mathcal{F}_{0}\right)$ for every $f \in \mathcal{H}$ (then $P$ is a Markov operator). Clearly, $U$ is a unitary dilation of $P$. Let $\left(u_{i}\right)_{i \in \mathbb{N}} \in c_{00}$ and define $f:=\sum_{i \in \mathbb{N}} u_{i} \varepsilon_{i} \in \mathcal{H}$. Assume moreover that $\left.\sum_{n \in \mathbb{N}}\left|a_{n}\right|\left(\sum_{k=0}^{n}\left|a_{k}\right|\right)\right)\left\|P^{n} f\right\|_{\mathcal{H}}^{2}<\infty$, or equivalently (by Lemma 44), $\sum_{n \in \mathbb{N}}\left(\sum_{k=0}^{n}\left|a_{k}\right|\right)^{2}\left\|U^{-n} P^{n} f-U^{-n-1} P^{n+1} f\right\|_{2}^{2}$. Notice that $P^{n} f=$ $\sum_{i \in \mathbb{N}} u_{i+n} \varepsilon_{-i}$ and that $\left\|U^{-n} P^{n} f-U^{-n-1} P^{n+1} f\right\|_{2}^{2}=u_{n}^{2}$. Moreover, $\left\|\sum_{n \in \mathbb{N}} a_{n} P^{n} f\right\|_{\mathcal{H}}^{2}=$ $\sum_{i \in \mathbb{N}}\left|\sum_{n \in \mathbb{N}} a_{n} u_{i+n}\right|^{2}$. Hence, $\sum_{i \in \mathbb{N}}\left|\sum_{n \in \mathbb{N}} a_{n} u_{i+n}\right|^{2} \leq C^{2} \sum_{n \in \mathbb{N}} b_{n}\left|u_{n}\right|^{2}$, i.e. (4) holds with $q=2$, and the proof is complete.

We shall now prove that Proposition 5 cannot be improved.

Definition 1. We say that a contraction $P$ on $\mathcal{H}$ is Ritt if $\sup _{n \in \mathbb{N}} n\left\|P^{n}-P^{n+1}\right\|<\infty$.

Proposition 7. Let $P$ be a contraction on $\mathcal{H}$. For every $0 \leq \alpha<1$, consider the following properties.

(i) The series $\sum_{n \in \mathbb{N}}(n+1)^{-\alpha} P^{n} f$ converges in $\mathcal{H}$;

(ii) $\sum_{n \in \mathbb{N}}(n+1)^{1-2 \alpha}\left\|P^{n} f\right\|_{\mathcal{H}}^{2}<\infty$.

Then, $(i i) \Rightarrow(i)$. If moreover $P$ is Ritt then $(i) \Rightarrow(i i)$. 
Remark. By [11], when $P$ is a positive operator on $L^{2}(m)$ then $(i)$ of the proposition implies that the series $\sum_{n \in \mathbb{N}}(n+1)^{-\alpha} P^{n} f$ converges $m$-almost everywhere and the associated maximal function is in $L^{2}$. The fact that $(i) \Rightarrow(i i)$ has been proved by Cohen, Cuny and Lin [10] using results from Arhancet and Le Merdy [2]) when $\alpha \in(0,1)$ and $P$ is a positive Ritt contraction of some $L^{2}(m)$ (there are also analogous results in $L^{p}$ in [10]).

Proof. The fact that $(i i) \Rightarrow(i)$ is a direct application of Proposition 5 Assume that $P$ is a Ritt operator and that $\sum_{n \in \mathbb{N}} \frac{P^{n} f}{(n+1)^{\alpha}}$ converges in $\mathcal{H}$.

We start with the case $0<\alpha<1$. By Proposition 4.6 of Cohen, Cuny and Lin 11] (see also their example (v) page 8), we have

$$
\sum_{n \geq 0} \frac{\left\|P f+\cdots+P^{2^{n}} f\right\|_{\mathcal{H}}^{2}}{2^{2 \alpha n}}<\infty
$$

Then, using (3) of Cohen, Cuny and Lin [10] combined with Lemma 13 below, we infer that $\sum_{n \geq 0} 2^{(2-2 \alpha) n}\left\|P^{2^{n}} f\right\|_{\mathcal{H}}^{2}<\infty$, which finishes the proof, in that case.

Assume now that $\alpha=0$. Let $g:=\sum_{n \in \mathbb{N}} P^{n} f$. Then, $f=(I-P) g$. Hence, by Theorem 8.1 of Le Merdy [24,

$$
\sum_{n \in \mathbb{N}} n\left\|P^{n} f\right\|_{\mathcal{H}}^{2}=\sum_{n \in \mathbb{N}} n\left\|P^{n}(I-P) g\right\|_{\mathcal{H}}^{2} \leq\|g\|_{\mathcal{H}},
$$

which is the desired result.

\section{Almost everywhere convergence of power series of $L^{2}$-contractions}

Once norm convergence has been proven, one may wonder, in the case where $\mathcal{H}=L^{2}(m)$, whether almost everywhere convergence holds. As mentionned in the remark following Proposition 7, for "regular" sequences, if $P$ is a positive contraction of $L^{2}(m)$ then norm convergence implies almost everywhere convergence. However, as we shall see below (see Proposition 10), there is no such result for contractions that are not positive. Let us mention that the almost everywhere convergence of power series (for regular $\left(a_{n}\right)_{n \in \mathbb{N}}$ ) for unitary or normal operators on $L^{2}(m)$ has been proven under conditions involving $\left(\left\|f+\ldots+P^{n} f\right\|_{\mathcal{H}}\right)_{n \in \mathbb{N}}$ in [23] and [13], see also [15] for $L^{p}$-versions.

Theorem 8. Let $\left(a_{n}\right)_{n \in \mathbb{N}} \in \mathbb{C}^{\mathbb{N}}$ be such that $N_{|\mathbf{a}|}$ be bounded on $\ell^{2}(\mathbb{N})$ where $|\mathbf{a}|=\left(\left|a_{n}\right|\right)_{n \in \mathbb{N}}$. Let $A_{n}:=\sum_{k=0}^{n}\left|a_{k}\right|$. Let $P$ be a contraction on $L^{2}(m)$. Let $f \in L^{2}(m)$ such that

$$
\sum_{n \geq 1}(\log (n+1))^{2} A_{2^{n+1}}^{2}\left\|P^{2^{n}} f\right\|_{L^{2}(m)}^{2}<\infty .
$$

Then, the series $\sum_{n \in \mathbb{N}} a_{n} P^{n} f$ converges $m$-almost everywhere and

$$
\sup _{N \geq 1}\left|\sum_{n=0}^{N} a_{n} P^{n} f\right| \in L^{2}(m) .
$$

Remark. A sufficient condition for (17) is the following

$$
\sum_{n \geq 1}(\log \log (n+3))^{2} \frac{A_{4 n}^{2}}{n+1}\left\|P^{n} f\right\|_{L^{2}(m)}^{2}<\infty .
$$


Proof. Let $N \in \mathbb{N}$. We have

$$
\max _{2^{N} \leq n \leq 2^{N+1}-1}\left|\sum_{k=2^{N}}^{n} a_{n} P^{k} f\right| \leq \sum_{k=2^{N}}^{2^{N+1}-1}\left|a_{k}\right|\left|P^{k} f\right| .
$$

Hence,

$$
\sum_{N \in \mathbb{N}}\left\|\max _{2^{N} \leq n \leq 2^{N+1}-1}\left|\sum_{k=2^{N}}^{n} a_{n} P^{k} f\right|\right\|_{L^{2}(m)}^{2} \leq \sum_{N \in \mathbb{N}} A_{2^{N+1}}^{2}\left\|P^{2^{N}} f\right\|_{L^{2}(m)}^{2}<\infty .
$$

In particular, it suffices to prove that $\left(\sum_{n=0}^{2^{N}} a_{n} P^{n} f\right)_{N \geq 0}$ converges and that $\sup _{N \geq 0}\left|\sum_{n=0}^{2^{N}} a_{n} P^{n} f\right| \in$ $L^{2}(m)$.

By (14), for every $q \geq p$, we have

$$
\left\|\sum_{n=2^{p}}^{2^{q}-1} a_{n} P^{n} f\right\|_{L^{2}(m)}^{2} \leq \sum_{n \in \mathbb{N}}\left(\sum_{k=2^{p}}^{2^{q}-1}\left|a_{k}\right| u_{n+k}\right)^{2} .
$$

Set $d(p, q):=\sum_{n \in \mathbb{N}}\left(\sum_{k=2^{p}}^{2^{q}-1}\left|a_{k}\right| u_{n+k}\right)^{2}$ and notice that $d$ is super-additive in the following sense: for every $m \geq l \geq k, d(k, l)+d(l, m) \leq d(k, m)$. By Proposition 2.2 of Cohen and Lin [12], there exists $C>0$, such that for every $n \geq 0$,

$$
\left\|\max _{2^{2^{n}} \leq m \leq 2^{2^{n+1}}-1}\left|\sum_{k=2^{2^{n}}}^{m} a_{k} P^{k} f\right|\right\|_{L^{2}(m)}^{2} \leq C(n+1)^{2} d\left(2^{n}, 2^{n+1}-1\right) .
$$

Assume that

$$
\sum_{n \geq 0}(n+1)^{2} d\left(2^{n}, 2^{n+1}-1\right)<\infty .
$$

Then, using (19) and Cauchy-Schwarz we see that

$$
\left(\sum_{n \in \mathbb{N}}\left\|\sum_{k=2^{2^{n}}}^{2^{2^{n+1}}-1} a_{k} P^{k} f\right\|_{L^{2}(m)}\right)^{2} \leq \sum_{n \in \mathbb{N}} \frac{1}{(n+1)^{2}} \sum_{n \in \mathbb{N}}(n+1)^{2} d\left(2^{n}, 2^{n+1}-1\right) .
$$

This finishes the proof, provided that we can show (20).

But, (20) reads

$$
\sum_{n \in \mathbb{N}} \sum_{\ell \geq 0}(\ell+1)^{2}\left(\sum_{k=2^{2^{\ell}}}^{2^{2^{\ell+1}}-1}\left|a_{k}\right| u_{n+k}\right)^{2}<\infty .
$$

Using that $\|\cdot\|_{\ell^{2}} \leq\|\cdot\|_{\ell^{1}}$, we infer that

$$
\begin{aligned}
\sum_{n \in \mathbb{N}} \sum_{\ell \geq 0}(\ell+1)^{2} & \left(\sum_{k=2^{2^{\ell}}}^{2^{2^{\ell+1}}-1}\left|a_{k}\right| u_{n+k}\right)^{2} \leq \sum_{n \in \mathbb{N}}\left(\sum_{k \geq 0}(\log \log (k+3))^{2}\left|a_{k}\right| u_{n+k}\right)^{2} \\
& \leq \sum_{n \in \mathbb{N}}\left(\sum_{k \geq 0}(\log \log (n+k+3))^{2}\left|a_{k}\right| u_{n+k}\right)^{2} .
\end{aligned}
$$


Then, proceeding as in the (end of the) proof of Proposition [5 we see that (20) holds provided that

$$
\sum_{n \in \mathbb{N}}(\log \log (n+3))^{2} A_{n}^{2} u_{n}^{2}<\infty,
$$

which follows from (17) using that $\left(A_{n}\right)_{n \in \mathbb{N}}$ is non-decreasing and that $\sum_{k=2^{n}}^{2^{n+1}-1} u_{k}^{2} \leq\left\|P^{2^{n}} f\right\|_{L^{2}(m)}^{2}$.

Corollary 9. Let $(X, \Sigma, \mu, \theta)$ be an ergodic dynamical system. Let $g \in L^{p}(\mu)$ for some $p>1$. There exists $\bar{X} \in \Sigma$ with $\mu(\bar{X})=1$ such that for every $x \in \bar{X}$, setting $\left(a_{n}\right)_{n \in \mathbb{N}}:=\left(g \circ \theta^{n}(x)\right)_{n \in \mathbb{N}}$ the following holds: for every $0 \leq \alpha<1$, every contraction $P$ on $L^{2}(m)$ and every $f \in L^{2}(m)$ such that

$$
\sum_{n \in \mathbb{N}}(\log \log (n+3))^{2}(n+1)^{1-2 \alpha}\left\|P^{n} f\right\|_{2}^{2}<\infty
$$

the sequence $\sum_{n \in \mathbb{N}} \frac{a_{n} P^{n} f}{(n+1)^{\alpha}}$ converges m-almost everywhere and the associated maximal function if in $L^{2}(m)$.

Proof. Let $(X, \Sigma, \mu, \theta)$ and let $g \in L^{p}(\mu)$. Let $\bar{X}$ be the set appearing in the example $(i v)$. Modifying $\bar{X}$ if necessary we may assume that $A_{n}=\left|a_{0}\right|+\ldots+\left|a_{n}\right| \leq K(x) n$, for some finite $K(x)>0$. Then, for every $x \in \bar{X},\left(g \circ \theta^{n}(x)\right)_{n \in \mathbb{N}}$ is good for the dominated weighted ergodic theorem. Applying Proposition 2, we see that, with $\mathbf{c}=\left(c_{n}\right)_{n \in \mathbb{N}}:=\left((n+1)^{-\alpha} f \circ \theta^{n}(x)\right)_{n \in \mathbb{N}}, N_{\mathbf{c}}$ is bounded on $\ell^{2}$. Set $C_{n}:=\sum_{k=0}^{n}\left|c_{k}\right|$ (we see $C_{n}$ as a function on $X$ ). By Theorem 8 (see the remark after the theorem), we are back to prove that $\sum_{n \geq 1}(\log \log (n+1))^{2} C_{4 n}^{2}\left\|P^{n} f\right\|_{2}^{2}<\infty$. But this follows our assumption (and an Abel summation) since $A_{n} \leq K(x) n$.

We shall now prove that Corollary 9 (and hence Theorem 8) is sharp.

Proposition 10. Let $0 \leq \alpha<1$. There exists an operator $P$ on some $L^{2}(m)$ and $f \in L^{2}(m)$ such that, for every $\varepsilon>0, \sum_{n \in \mathbb{N}}(\log \log (n+3))^{2-\varepsilon}(n+1)^{1-2 \alpha}\left\|P^{n} f\right\|_{2}^{2}<\infty$ and the series $\sum_{n \in \mathbb{N}}(n+1)^{-\alpha} P^{n}(f)$ diverges m-almost everywhere.

Remarks. The proof is related to some arguments of Gaposhkin [22] and makes use of a counterexample by Tandori in the theory of orthogonal series. The construction of the operator $P$ is related to the construction of the operator used in the proof of Proposition 6 . Actually, the operator $P$ used in the proof is a one-sided shift, hence is a co-isometry which prevent it from being Ritt. This raises the question whether it is possible to find a Ritt contraction satisfying the conclusion of the Proposition.

Proof. Let $\left(\varepsilon_{n}\right)_{n \in \mathbb{N}}$ be an orthormal system on some $L^{2}(m)$ that we shall specify later. We define an operator $P$ on $\overline{\operatorname{Vect}\left\{\varepsilon_{n}: n \in \mathbb{N}\right\}}$ as follows. For every $f=\sum_{n \in \mathbb{N}} c_{n} \varepsilon_{n}$ let $P f:=$ $\sum_{n \in \mathbb{N}} c_{n+1} \varepsilon_{n}$. One may extend $P$ to the whole $L^{2}(m)$ as one please.

For every $n \in \mathbb{N}$, let $c_{n}:=\frac{1}{(n+1)^{3 / 2-\alpha} \sqrt{\log (n+2) \log \log (n+3)^{3 / 2}}}$ and define $f$ as above.

We have

$$
\sum_{n=0}^{2^{N}}(n+1)^{-\alpha} P^{n} f=\sum_{n=0}^{2^{N}} \sum_{k \geq 0}(n+1)^{-\alpha} c_{k+n} \varepsilon_{k}
$$

We first prove that

$$
\sum_{n=0}^{2^{N}} \sum_{k \geq 2^{N}+1}(n+1)^{-\alpha} c_{k+n} \varepsilon_{k}:=v_{N} \underset{N \rightarrow \infty}{\longrightarrow} 0 \quad m \text {-a.e. }
$$


We have

$$
\left\|v_{N}\right\|_{L^{2}(m)}^{2}=\sum_{k \geq 2^{N}+1}\left(\sum_{n=0}^{2^{N}}(n+1)^{-\alpha} c_{k+n}\right)^{2} \leq C 2^{2 N(1-\alpha)} \sum_{k \geq 2^{N}+1} c_{k}^{2} \leq \frac{C^{\prime}}{N(\log (N+1))^{3}} .
$$

Hence, $\sum_{N \in \mathbb{N}}\left\|v_{N}\right\|_{L^{2}(m)}^{2}<\infty$ and (21) holds.

Next, we prove that

$$
\sum_{k=0}^{2^{N}} \sum_{n \geq 2^{N}+1}(n+1)^{-\alpha} c_{k+n} \varepsilon_{k}:=w_{N} \underset{N \rightarrow \infty}{\longrightarrow} 0 \quad m \text {-a.e. }
$$

We have

$$
\left\|v_{N}\right\|_{L^{2}(m)}^{2}=\sum_{k=0}^{2^{N}}\left(\sum_{n \geq 2^{N}+1}(n+1)^{-\alpha} c_{k+n}\right)^{2} \leq C 2^{N}\left(\sum_{n \geq 2^{N}+1}(n+1)^{-\alpha} c_{n}\right)^{2} \leq \frac{C^{\prime}}{N(\log (N+1))^{3}} .
$$

Hence, $\sum_{N \in \mathbb{N}}\left\|w_{N}\right\|_{L^{2}(m)}^{2}<\infty$ and (22) holds.

Combining those first results, we see that we are back to finding $\left(\varepsilon_{n}\right)_{n \in \mathbb{N}}$ such that $\left(\sum_{k=0}^{2^{N}} \sum_{n>0}(n+\right.$ $\left.1)^{-\alpha} c_{n+k} \varepsilon_{k}\right)_{N \in \mathbb{N}}$ diverges $m$-almost everywhere.

For every $k \geq 1$, define

$$
\begin{gathered}
\alpha_{k}:=\int_{0}^{\infty} \frac{d x}{x^{\alpha}(x+k)^{3 / 2-\alpha}\left(\log (x+k)(\log \log (x+k+2))^{3}\right)^{1 / 2}} \\
=k^{-1 / 2} \int_{0}^{\infty} \frac{d u}{u^{\alpha}(u+1)^{3 / 2-\alpha}\left(\log (k u+k)(\log \log (k u+k+2))^{3}\right)^{1 / 2}} .
\end{gathered}
$$

Hence, $\left(\sqrt{k} \alpha_{k}\right)_{k \geq 1}$ is non-increasing. Moreover, it is not hard to see that the series

$$
\sum_{k \geq 0}\left(\sum_{n \geq 0}(n+1)^{-\alpha} c_{n+k}-\alpha_{k}\right) \varepsilon_{k}
$$

converges $m$-almost everywhere.

Then, by a result of Tandori, see Theorem 2.9.1 page 143 of Alexits [1] (combined with Theorem 2.7.3 page 120) there exists an orthonormal system $\left(\varepsilon_{n}\right)_{n \in \mathbb{N}}$ such that the $\left(\sum_{n=0}^{2^{N}} \alpha_{n} \varepsilon_{n}\right)_{N \in \mathbb{N}}$ diverges $m$-a.e., and the proof is complete.

\section{Extensions, problems}

Recall that an operator $T$ on $\mathcal{H}$ is said to be similar to a contraction if there exists a continuous invertible operator $V$ from $\mathcal{H}$ onto $\mathcal{H}$ such that $\left\|V T V^{-1}\right\| \leq 1$, i.e. such that $V T V^{-1}$ be a contraction.

Clearly, all the results from section 3 extend to operators that are similar to a contraction. Now, when $\mathcal{H}=L^{2}(m)$, it can be checked that all the results from section 4 also hold for operators that are similar to a contraction, even though the operator $V$ in the definition need not be positive.

The most general class of operators on $\mathcal{H}$ to which one may hope to extend Proposition 7 is the class of power bounded operators. Recall that an operator $P$ on $\mathcal{H}$ is said to be power bounded if $\sup _{n \in \mathbb{N}}\left\|P^{n} f\right\|<\infty$. However, we shall see that this extension is not possible, even if we ask the operator to be Ritt. 
The next proposition is a reformulation of Proposition 8.2 of [24].

Proposition 11. There exists a Ritt power bounded operator $T$ on some Hilbert space $\mathcal{H}$ such that, taking $\alpha=0$, (ii) of Proposition 7 does not imply $(i)$.

Proof. Let $T$ be the operator defined in Proposition 8.2 of Le Merdy [24]. Then, $T$ is power bounded and Ritt and has no fixed point. Assume that for every $f \in \mathcal{H}$, the condition $\sum_{n \in \mathbb{N}} n\left\|T^{n} f\right\|_{\mathcal{H}}^{2}<\infty$ implies that $\sum_{n \in \mathbb{N}} T^{n} f$ converges in $\mathcal{H}$. Then, arguing as in the proof of Proposition 6, there exists $C>0$ such that $\left\|\sum_{n \in \mathbb{N}} T^{n} f\right\|_{\mathcal{H}} \leq C \sum_{n \in \mathbb{N}} n\left\|T^{n} f\right\|_{\mathcal{H}}^{2}$, whenever the right-hand side converges.

It follows from the proof of Proposition 8.2 of 24 that for every $g \in \mathcal{H}, \sum_{n \in \mathbb{N}} n \|(I-$ $T) T^{n} g \|_{\mathcal{H}}^{2}<\infty$. Hence, for every $g \in \mathcal{H}$, the series $\sum_{n \in \mathbb{N}} T^{n}(I-T) g$ converges in $\mathcal{H}$, say to $h$. Then, $\|h\| \leq C \sum_{n \in \mathbb{N}} n\left\|(I-T) T^{n} g\right\|_{\mathcal{H}}^{2}$ and $(I-T) h=(I-T) g$. Since $T$ has no fixed point, we infer that $g=h$ and $\|g\| \leq C \sum_{n \in \mathbb{N}} n\left\|(I-T) T^{n} g\right\|_{\mathcal{H}}^{2}$ for every $g \in \mathcal{H}$. But it is proved in 24] that this cannot hold.

We now give an extension of Corollary 9 to the case where $\alpha=1$.

Proposition 12. Let $P$ be a contraction on $L^{2}(m)$. Let $f \in L^{2}(m)$ be such that

$$
\sum_{n \in \mathbb{N}} \frac{\log (n+1)}{n+1}\left\|P^{n} f\right\|_{2}^{2}<\infty \text {. }
$$

Then, $\sum_{n \in \mathbb{N}} \frac{P^{n} f}{n+1}$ converges in $L^{2}(m)$. If moreover

$$
\sum_{n \in \mathbb{N}} \frac{\log (n+1)(\log \log \log (n+9))^{2}}{n+1}\left\|P^{n} f\right\|_{2}^{2}<\infty
$$

then, $\sum_{n \in \mathbb{N}} \frac{P^{n} f}{n+1}$ converges m-almost everywhere and the associated maximal function is in $L^{2}(m)$.

Proof. The norm convergence follows easily from Proposition 5. We now give the main argument for the proof of the almost everywhere convergence. Then, the rest of the proof is similar to that of Proposition 8, Assume (23). We have

$$
\left\|\sum_{k=2^{2^{n}}}^{2^{2^{n+1}-1}} \frac{\left|P^{k} f\right|}{k}\right\|_{L^{2}(m)}^{2} \leq C 2^{2 n}\left\|P^{2^{2^{n}}} f\right\|_{L^{2}(m)}^{2} .
$$

It is not hard to see that (23) implies that $\sum_{n \geq 0} 2^{2 n}\left\|P^{2^{2^{n}}}\right\|_{L^{2}(m)}^{2}<\infty$. Hence it suffices to prove the desired convergence of the series along the sequence $\left(2^{2^{n}}\right)_{n \in \mathbb{N}}$.

A natural question is the following : does there exist analogous results to, say, Proposition 7 for contractions of $L^{p}$ spaces. For instance, by Cohen, Cuny and Lin [10, if $P$ is a positive Ritt contraction of some $L^{p}, 1<p \leq 2$ then the condition $\sum_{n \in \mathbb{N}}(n+1)^{1-p \alpha}\left\|P^{n} f\right\|_{p}^{p}<\infty$ is sufficient for the convergence in $L^{p}$ of $\sum_{n \in \mathbb{N}}(n+1)^{-\alpha} P^{n} f$ (and the a.e. convergence holds as well). The approach used in the present paper partially work for Markov operators. However, it does not seem to allow one to extend the results of [10] to Markov operators. It would be interesting either to prove that $L^{p}$ extensions are possible or to find an example where it cannot. 


\section{APPENDIX A.}

We made use of the following lemma which is related to Lemma 2.7 of Peligrad and Utev 27.

Lemma 13. Let $\left(V_{n}\right)_{n \geq 1}$ be a nonnegative subadditive sequence (i.e. $V_{n+m} \leq V_{n}+V_{m}$ for every $m, n \geq 1$ ). Then, for every $q \geq 1$ and every $p>1$, there exists $C>0$ such that $\sum_{n \geq 1} \frac{\max _{1 \leq i \leq n} V_{n}^{q}}{n^{p}} \leq C \sum_{n \geq 0} \frac{V_{2}^{q}}{2^{n p}}$.

Proof. The proof basically follows the arguments to prove Lemma 4.1 of [27. We start with the following basic (dyadic) decomposition. For every $r \geq 0$, and every $2^{r} \leq n \leq 2^{r+1}-1$, we have, by an easy induction,

$$
V_{n} \leq V_{n-2^{r}}+V_{2^{r}} \leq \sum_{k=0}^{r} V_{2^{k}}
$$

Hence,

$$
\sum_{n \geq 1} \frac{\max _{1 \leq i \leq n} V_{n}^{q}}{n^{p}} \leq \sum_{r \geq 0} \sum_{n=2^{r}}^{2^{r+1}-1} \frac{\max _{1 \leq i \leq n} V_{n}^{q}}{n^{p}} \leq \sum_{r \geq 0} \frac{1}{2^{(p-1) r}}\left(\sum_{k=0}^{r} V_{2^{k}}\right)^{q}
$$

When $q=1$ the result follows. Assume that $q>1$. Let $0<\varepsilon<p-1$. Using Hölder's inequality (with $1 / q+1 / q^{\prime}=1$ ) 1 , we have

$$
\sum_{n \geq 1} \frac{\max _{1 \leq i \leq n} V_{n}^{q}}{n^{p}} \leq \sum_{r \geq 0} \frac{1}{2^{(p-1) r}}\left(\sum_{j=0}^{r} 2^{k \varepsilon q^{\prime} q}\right)^{q / q^{\prime}}\left(\sum_{k=0}^{r} 2^{-k \varepsilon} V_{2^{k}}^{q}\right) \leq C \sum_{n \geq 0} \frac{V_{2^{n}}^{q}}{2^{n p}} .
$$

\section{REFERENCES}

[1] G. Alexits, Convergence problems of orthogonal series, Translated from the German by I. Földer. International Series of Monographs in Pure and Applied Mathematics, Vol. 20 Pergamon Press, New York-OxfordParis 1961 ix $+350 \mathrm{pp}$

[2] C. Arhancet and C. Le Merdy, Dilation of Ritt operators on $L^{p}$-spaces, Israel J. Math. 201 (2014), no. 1, 373-414.

[3] A. Bellow and V. Losert, The weighted pointwise ergodic theorem and the individual ergodic theorem along subsequences, Trans. Amer. Math. Soc. 288 (1985), no. 1, 307-345.

[4] G. Bennett, Inequalities complimentary to Hardy, Q. J. Math., Oxf. II. (1998) Ser. 49, no.196, 395-432.

[5] D. Borwein, Nörlund operators on $l_{p}$, Canad. Math. Bull. 36 (1993), no. 1, 8-14.

[6] D. Borwein and F. P. Cass, Nörlund matrices as bounded operators on $\ell^{p}$, Arch. Math. (Basel) 42 (1984), no. $5,464-469$.

[7] J. Bourgain, An approach to pointwise ergodic theorems, Geometric aspects of functional analysis (1986/87), 204-223, Lecture Notes in Math., 1317, Springer, Berlin, 1988.

[8] J. Bourgain, Temps de retour pour les systmes dynamiques, C. R. Acad. Sci. Paris Sr. I Math. 306 (1988), no. $12,483-485$.

[9] J. Bourgain, Pointwise ergodic theorems for arithmetic sets. With an appendix by the author, Harry Furstenberg, Yitzhak Katznelson and Donald S. Ornstein, Inst. Hautes études Sci. Publ. Math. No. 69 (1989), 5-45.

[10] G. Cohen, C. Cuny and M. Lin, Almost everywhere convergence of powers of some positive $L_{p}$ contractions, J. Math. Anal. Appl. 420 (2014), no. 2, 1129-1153.

[11] G. Cohen, C. Cuny and M. Lin, On the convergence of power series of $L_{p}$-contractions, accepted for publication in Banach Center Volume.

[12] G. Cohen and M. Lin, Extensions of the Menchoff-Rademacher theorem with applications to ergodic theory, Probability in mathematics. Israel J. Math. 148 (2005), 41-86.

[13] C. Cuny, Pointwise ergodic theorems with rate with applications to limit theorems for stationary processes, Stoch. Dyn. 11 (2011), no. 1, 135-155. 
[14] C. Cuny, Norm convergence of some power series of operators in $L^{p}$ with applications in ergodic theory, Stud. Math. 200 (2010), no. 1, 1-29.

[15] C. Cuny, Almost everywhere convergence of generalized ergodic transforms for invertible power-bounded operators in Lp, Colloq. Math. 124 (2011), no. 1, 61-77.

[16] C. Cuny and M. Lin, Limit theorems for Markov chains by the symmetrization method, J. Math. Anal. Appl. 434 (2016), no. 1, 52-83.

[17] C. Cuny and M. Weber, Ergodic theorems with arithmetical weights, Israel J. Math. 217 (2017), no. 1, 139-180.

[18] B. Delyon and F. Delyon, Generalization of von Neumann's spectral sets and integral representation of operators, Bull. Soc. Math. France 127 (1999), no. 1, 25-41.

[19] C. Demeter, Improved range in the return times theorem, Canad. Math. Bull. 55 (2012), no. 4, 708-722.

[20] Y. Derriennic and M. Lin, Fractional Poisson equations and ergodic theorems for fractional coboundaries, Israel J. Math. 123 (2001), 93-130.

[21] C. Demeter, M. Lacey, T. Tao and C. Thiele, Breaking the duality in the return times theorem, Duke Math. J. 143 (2008), no. 2, 281-355.

[22] V. F. Gaposhkin, Criteria for the strong law of large numbers for classes of stationary processes and homogeneous random fields, Dokl. Akad. Nauk SSSR 223 (1975), no. 5, 1044-1047.

[23] V.F. Gaposhkin, Spectral criteria for existence of generalized ergodic transforms, Theory Probab. Appl. 41 (1996), no.2, 247-264 ; translation from Teor. Veroyatn. Primen. 41 (1996), no.2, 251-271.

[24] C. Le Merdy, $H^{\infty}$ functional calculus and square function estimates for Ritt operators, Rev. Mat. Iberoam. 30 (2014), no. 4, 1149-1190.

[25] F. Riesz and B. Sz.-Nagy, Functional analysis. Translated from the second French edition by Leo F. Boron. Reprint of the 1955 original. Dover Books on Advanced Mathematics. Dover Publications, Inc., New York, 1990.

[26] J. J. Shäffer, On unitary dilations of contractions, Proc. Amer. Math. Soc. 6, (1955). 322

[27] M. Peligrad and S. Utev, A new maximal inequality and invariance principle for stationary sequences, Ann. Probab. 33 (2005), no. 2, 798-815.

[28] D. Volný, Martingale-coboundary Representation for stationary random fields, preprint.

[29] M. Weber, Dynamical systems and processes. IRMA Lectures in Mathematics and Theoretical Physics, 14. European Mathematical Society (EMS), Zrich, 2009. xii+761 pp.

[30] M. Wierdl, Pointwise ergodic theorem along the prime numbers, Israel J. Math. 64 (1988), no. 3, 315-336 (1989).

Université de la Nouvelle-CAlédonie Equipe ERIM, B.P. 4477, F-98847 Noumea Cedex

E-mail address: cuny@univ-nc.nc

IRMA, 10 rue du Général Zimmer, 67084 Strasbourg Cedex, France

E-mail address: michel.weber@math.unistra.fr 